Bull. Austral. Math. Soc.

$43 \mathrm{~A} 30,43 \mathrm{~A} 80$.

Vol. 76 (2007) [471-478]

\title{
AN ANALOGUE OF BEURLING'S THEOREM FOR THE HEISENBERG GROUP
}

\author{
Jizheng Huang and Heping LiU
}

In this paper, we prove an analogue of Beurling's theorem on the Heisenberg group. Then we derive some other versions of the uncertainty principle.

\section{INTRODUCTION}

The uncertainty principle states that a function and its Fourier transform cannot simultaneously decay very rapidly. This principle has several versions which were proved by Hardy, Morgan, Cowling-Price and Gelfand-Shilov et cetera (see [6, 18]). A more general version of uncertainty principle, which is called Beurling's theorem, has been proved by Hörmander [7] and generalised by Bonami, Demange and Jaming [4] as follows:

THEOREM 1. Let $f \in L^{2}\left(\mathbb{R}^{n}\right)$ and $d \geqslant 0$. Then

$$
\int_{\mathbf{R}^{n}} \int_{\mathbf{R}^{n}} \frac{|f(x)||\hat{f}(y)|}{(1+|x|+|y|)^{d}} e^{|x||y|} d x d y<\infty
$$

implies that

$$
f(x)=P(x) e^{-\langle A x, x)},
$$

where $A$ is a real positive definite symmetric matrix and $P$ is a polynomial of degree $<(d-n / 2)$. In particular, $f=0$ when $d \leqslant n$.

Beurling's theorem has been extended to different settings (see $[11,12,19]$ ). For the Heisenberg group and the other nilpotent Lie groups, some theorems of Hardy type, Cowling-Price type and Morgan type have been proved by various authors (see $[1,2,3$, $8,9,10,13,16,17])$. These versions of uncertainty principle are also established for the semisimple Lie groups or the symmetric spaces. We refer the reader to $[18,19]$ and the references therein.

The goal of this paper is to prove an analogue of Beurling's theorem for the Heisenberg group. As pointed out in [19], there are some difficulties in extending Beurling's theorem to a general setting. It should be pointed out that Thangavelu (see $[16,17]$ )

Received 5th June, 2007

Supported by the National Natural Science Foundation of China (No. 10371004) and the Specialised Research Fund for the Doctoral Program of Higher Education of China (No. 20030001107).

Copyright Clearance Centre, Inc. Serial-fee code: 0004-9727/07 \$A2.00+0.00. 
gives a heat kernel version of Hardy's theorem on the Heisenberg group. The heat kernel version of Beurling's theorem on the Heisenberg group is still open.

This paper is organised as follows. In the rest of this section, we recall some basic facts about the Heisenberg group. The main result is proved in the next section. In the last section we derive some other versions of uncertainty principle including some known results mentioned above.

As the basic references for the Heisenberg group we refer the reader to $[5,15]$ or [18]. The Heisenberg group $\mathbb{H}^{n}$ is a Lie group with the underlying manifold $\mathbb{C}^{n} \times \mathbb{R}$ and the group law

$$
(z, t)(w, s)=\left(z+w, t+s+\frac{1}{2} \operatorname{Im}(z \cdot \bar{w})\right) .
$$

The Haar measure on $\mathbb{H}^{n}$ coincides with the Lebesgue measure $d z d t$ on $\mathbb{C}^{n} \times \mathbb{R}$. For $\lambda \in \mathbb{R}^{*}=\mathbb{R} \backslash\{0\}$, let $\pi_{\lambda}(z, t)$ denote the Schrödinger representation of $\mathbb{H}^{n}$, which acts on $L^{2}\left(\mathbb{R}^{n}\right)$ by

$$
\pi_{\lambda}(z, t) \varphi(\xi)=e^{i \lambda t} e^{i \lambda(x \cdot \xi+(1 / 2) x \cdot y)} \varphi(\xi+y),
$$

where $z=x+i y$. The group Fourier transform of a function $f \in L^{1}\left(\mathbb{H}^{n}\right)$ is the operator valued function defined by

$$
\widehat{f}(\lambda)=\int_{\mathbb{E}^{n}} f(z, t) \pi_{\lambda}(z, t) d z d t .
$$

We note that

$$
\widehat{f}(\lambda)=\int_{\mathbb{C}^{n}} f^{\lambda}(z) \pi_{\lambda}(z) d z,
$$

where $\pi_{\lambda}(z)=\pi_{\lambda}(z, 0)$ and

$$
f^{\lambda}(z)=\int_{-\infty}^{\infty} f(z, t) e^{i \lambda t} d t
$$

is the inverse Fourier transform of $f$ in the $t$-variable.

Let $\mathcal{S}_{2}$ denote the Hilbert space of Hilbert-Schmidt operators on $L^{2}\left(\mathbb{R}^{n}\right)$ with the inner product $(T, S)=\operatorname{tr}\left(S^{*} T\right)$. Let $d \mu(\lambda)=(2 \pi)^{-n-1}|\lambda|^{n} d \lambda$ and let $L^{2}\left(\mathbb{R}^{*}, \mathcal{S}_{2}, d \mu\right)$ be the space of functions on $\mathbb{R}^{*}$ taking values in $\mathcal{S}_{2}$ and square integrable with respect to $d \mu$. The Plancherel theorem on $\mathbb{H}^{n}$ states that the group Fourier transform is extended to an isometric isomorphism from $L^{2}\left(\mathbb{H}^{n}\right)$ onto $L^{2}\left(\mathbb{R}^{*}, \mathcal{S}_{2}, d \mu\right)$; that is,

$$
\int_{\mathbf{B}^{n}}|f(z, t)|^{2} d z d t=\int_{\mathbf{R}}\|\hat{f}(\lambda)\|_{H S}^{2} d \mu(\lambda)
$$

where $\|\hat{f}(\lambda)\|_{H S}$ denotes the Hilbert-Schmidt norm of $\widehat{f}(\lambda)$ and satisfies

$$
|\lambda|^{n}\|\widehat{f}(\lambda)\|_{H S}^{2}=(2 \pi)^{n} \int_{\mathbf{C}^{n}}\left|f^{\lambda}(z)\right|^{2} d z .
$$

Now we introduce the special Hermite functions (see [14]). Let $H_{k}(t)$ be the Hermite polynomials defined by

$$
H_{k}(t)=(-1)^{k} e^{t^{2}} \frac{d^{k}}{d t^{k}}\left(e^{-t^{2}}\right), \quad k=0,1,2, \ldots
$$


The normalised Hermite functions are defined by

$$
h_{k}(t)=\left(2^{k} \sqrt{\pi} k !\right)^{-1 / 2} H_{k}(t) e^{-(1 / 2) t^{2}} .
$$

The $n$-dimensional Hermite functions $\Phi_{\alpha}$ are defined by taking the tensor products; that is,

$$
\Phi_{\alpha}(x)=\prod_{j=1}^{n} h_{\alpha_{j}}\left(x_{j}\right), \quad \alpha \in \mathbb{N}^{n} .
$$

It is well known that $\left\{\Phi_{\alpha}: \alpha \in \mathbb{N}^{n}\right\}$ form an orthonormal basis for $L^{2}\left(\mathbb{R}^{n}\right)$. Then we define the special Hermite functions $\Phi_{\alpha, \beta}$ by

$$
\begin{aligned}
\Phi_{\alpha, \beta}(z) & =(2 \pi)^{-n / 2}\left(\pi_{1}(z) \Phi_{\alpha}, \Phi_{\beta}\right) \\
& =(2 \pi)^{-n / 2} \int_{\mathbf{R}^{n}} e^{i x \cdot \xi} \Phi_{\alpha}\left(\xi+\frac{y}{2}\right) \Phi_{\beta}\left(\xi-\frac{y}{2}\right) d \xi
\end{aligned}
$$

Obviously,

$$
\left|\Phi_{\alpha, \beta}(z)\right| \leqslant(2 \pi)^{-n / 2}
$$

Also we have

LEMMA 1. The special Hermite functions $\left\{\Phi_{\alpha, \beta}: \alpha, \beta \in \mathbb{N}^{n}\right\}$ form an orthonormal basis for $L^{2}\left(\mathbb{C}^{n}\right)$.

\section{AN ANALOgue of BeURLing's theorem}

In this section, we prove an analogue of Beurling's theorem for the Heisenberg group. The result is stated as follows.

THEOREM 2. Let $f \in L^{2}\left(\mathbb{H}^{n}\right)$ and $d \geqslant 0$. Suppose that

$$
\int_{\mathbf{R}^{n}} \int_{\mathbb{R}} \frac{|f(z, t)|\|\hat{f}(\lambda)\|_{H S}}{(1+|t|+|\lambda|)^{d}} e^{|t||\lambda|} d \mu(\lambda) d(z, t)<\infty .
$$

Then

$$
f(z, t)=e^{-a t^{2}}\left(\sum_{j=0}^{m} \varphi_{j}(z) t^{j}\right)
$$

where $a$ is a positive constant, $\varphi_{j} \in L^{2}\left(\mathbb{C}^{n}\right)$ and $m<(d-((n / 2)+1)) / 2$. In particular, $f(z, t)=\mathrm{e}^{-a t^{2}} f(z, 0)$ when $d \leqslant n / 2+3$ and $f=0$ when $d \leqslant(n / 2)+1$.

ProOF: First we prove that $f \in L^{1}\left(\mathbb{H}^{n}\right)$. Let

$$
A=\left\{\lambda:\|\hat{f}(\lambda)\|_{H S} \neq 0\right\} \text {. }
$$


We may assume that $A$ has positive Lebesgue measure. Otherwise, $f=0$. By (4), there exists $\lambda_{0} \neq 0$ such that

$$
\int_{\mathbf{B}^{n}} \frac{|f(z, t)|}{\left(1+|t|+\left|\lambda_{0}\right|\right)^{d}} e^{|t|\left|\lambda_{0}\right|} d(z, t)<\infty
$$

Because there exists $C>0$ such that $\left(1+|t|+\left|\lambda_{0}\right|\right)^{d} \leqslant C e^{|t|\left|\lambda_{0}\right|}$,

$$
\int_{\mathbb{H}^{n}}|f(z, t)| d(z, t) \leqslant C \int_{\mathbf{H}^{n}} \frac{|f(z, t)|}{\left(1+|t|+\left|\lambda_{0}\right|\right)^{d}} e^{|t|\left|\lambda_{0}\right|} d(z, t)<\infty .
$$

Therefore, $f \in L^{1}\left(\mathbb{H}^{n}\right)$.

In view of (2) and Lemma 1 , we have

$$
|\lambda|^{n}\|\widehat{f}(\lambda)\|_{H S}^{2}=(2 \pi)^{n} \int_{\mathbb{C}^{n}}\left|f^{\lambda}(z)\right|^{2} d z=(2 \pi)^{n} \sum_{\alpha, \beta}\left|\left(f^{\lambda}, \Phi_{\alpha, \beta}\right)\right|^{2} .
$$

Set

$$
g_{\alpha, \beta}(t)=\int_{\mathbb{C}^{n}} f(z, t) \overline{\Phi_{\alpha, \beta}}(z) d z
$$

By (3),

$$
\left|g_{\alpha, \beta}(t)\right| \leqslant(2 \pi)^{-n / 2} \int_{\mathbf{C}^{n}}|f(z, t)| d z
$$

By (5),

$$
\left|\widehat{g_{\alpha, \beta}}(\lambda)\right|=\left|\left(f^{-\lambda}, \Phi_{\alpha, \beta}\right)\right| \leqslant(2 \pi)^{-n / 2}|\lambda|^{n / 2}\|\widehat{f}(-\lambda)\|_{H S} .
$$

For any $\alpha, \beta, \alpha^{\prime}, \beta^{\prime} \in \mathbb{N}^{n}$, it follows from (7), (8) and (4) that

$$
\begin{aligned}
\int_{\mathbb{R}} \int_{\mathbb{R}} \frac{\left|g_{\alpha, \beta}(t)\right|\left|\widehat{g_{\alpha^{\prime}, \beta^{\prime}}}(\lambda)\right|}{(1+|\lambda|+|t|)^{d}} & e^{|t||\lambda|}|\lambda|^{n / 2} d t d \lambda \\
& \leqslant 2 \pi \int_{\mathbf{B}^{n}} \int_{\mathbf{R}} \frac{|f(z, t)||| \widehat{f}(\lambda) \|_{H S}}{(1+|t|+|\lambda|)^{d}} e^{|t| \lambda \mid} d \mu(\lambda) d(z, t)<\infty .
\end{aligned}
$$

Now we prove

$$
\int_{\mathbf{R}} \int_{\mathbf{R}} \frac{\left|g_{\alpha, \beta}(t)\right|\left|\widehat{g_{\alpha^{\prime}, \beta^{\prime}}}(\lambda)\right|}{(1+|t|+|\lambda|)^{d}} e^{|t||\lambda|} d t d \lambda<\infty .
$$

Given $\alpha, \beta \in \mathbb{N}^{n}$, we define

$$
M(\lambda)=\int_{\mathbf{R}} \frac{\left|g_{\alpha, \beta}(t)\right|}{(1+|t|+|\lambda|)^{d}} e^{|t||\lambda|} d t
$$


It is concluded from (9) that there exists $\lambda_{0} \neq 0$ such that $M\left(\lambda_{0}\right)<\infty$. When $a>d$, the function $F(x)=(1+a+x)^{-d} e^{a x}$ is increasing on $[0, \infty)$. Hence for $|\lambda| \leqslant\left|\lambda_{0}\right|$,

$$
\begin{aligned}
M(\lambda) & =\int_{\mathbf{R}} \frac{\left|g_{\alpha, \beta}(t)\right|}{(1+|t|+|\lambda|)^{d}} e^{|t||\lambda|} d t \\
& \leqslant e^{d\left|\lambda_{0}\right|} \int_{|t| \leqslant d}\left|g_{\alpha, \beta}(t)\right| d t+\int_{|t|>d} \frac{\left|g_{\alpha, \beta}(t)\right|}{\left(1+|t|+\left|\lambda_{0}\right|\right)^{d}} e^{|t|\left|\lambda_{0}\right|} d t \\
& \leqslant e^{d\left|\lambda_{0}\right|}\|f\|_{L^{1}}+M\left(\lambda_{0}\right)<\infty .
\end{aligned}
$$

Note that $\left|\widehat{g_{\alpha^{\prime}, \beta^{\prime}}}(\lambda)\right| \leqslant(2 \pi)^{-n / 2}\|f\|_{L^{1}}$. From (9) and (11) we obtain

$$
\begin{aligned}
& \int_{\mathbb{R}} \int_{\mathbf{R}} \frac{\left|g_{\alpha, \beta}(t)\right|\left|\widehat{g_{\alpha^{\prime}, \beta^{\prime}}}(\lambda)\right|}{(1+|\lambda|+|t|)^{d}} e^{|t||\lambda|} d t d \lambda \\
& \quad \leqslant\left|\lambda_{0}\right|^{-n / 2} \int_{|\lambda|>\left|\lambda_{0}\right|} \int_{\mathbf{R}} \frac{\left|g_{\alpha, \beta}(t)\right|\left|\widehat{g_{\alpha^{\prime}, \beta^{\prime}}}(\lambda)\right|}{(1+|\lambda|+|t|)^{d}} e^{|t| \lambda \mid}|\lambda|^{n / 2} d t d \lambda+\int_{|\lambda| \leqslant\left|\lambda_{0}\right|} M(\lambda)\left|\widehat{g_{\alpha^{\prime}, \beta^{\prime}}}(\lambda)\right| d \lambda \\
& \quad<\infty .
\end{aligned}
$$

This proves (10). In particular, for any $\alpha, \beta \in \mathbb{N}^{n}$,

$$
\int_{\mathbb{R}} \int_{\mathbb{R}} \frac{\left|g_{\alpha, \beta}(t)\right|\left|\widehat{g_{\alpha, \beta}}(\lambda)\right|}{(1+|\lambda|+|t|)^{d}} e^{|t| \lambda \mid} d t d \lambda<\infty
$$

By Theorem 1,

$$
g_{\alpha, \beta}(t)=P_{\alpha, \beta}(t) e^{-a_{\alpha, \beta} t^{2}},
$$

where $P_{\alpha, \beta}$ are polynomials, $a_{\alpha, \beta}$ are positive constants. If $\operatorname{deg} P_{\alpha, \beta} \geqslant(d-((n / 2)+1)) / 2$, then (9) is false. So we have $\operatorname{deg} P_{\alpha, \beta}<(d-((n / 2)+1)) / 2$.

If $a_{\alpha, \beta} \neq a_{\alpha^{\prime}, \beta^{\prime}}$ for some $\alpha, \beta, \alpha^{\prime}, \beta^{\prime} \in \mathbb{N}^{n}$, then (10) will be false. So $a_{\alpha, \beta}=a$ are independent of $\alpha, \beta$. Let

$$
P_{\alpha, \beta}(t)=\sum_{j=0}^{m} C_{j, \alpha, \beta} t^{j} .
$$

Then

$$
f(z, t)=e^{-a t^{2}}\left(\sum_{j=0}^{m} \varphi_{j}(z) t^{j}\right)
$$

Where

$$
\varphi_{j}(z)=\sum_{\alpha, \beta} C_{j, \alpha, \beta} \Phi_{\alpha, \beta}(z) \in L^{2}\left(\mathbb{C}^{n}\right) .
$$

The proof of Theorem 2 is completed. 


\section{SOME OTHER VERSIONS OF THE UNCERTAINTY PRINCIPLE}

In this section we drive some other versions of uncertainty principle.

The following uncertainty principle of Gelfand-Shilov type is a direct consequence of Theorem 2.

COROLlaRY 1. Let $d \geqslant 0$ and assume that $f \in L^{2}\left(\mathbb{H}^{n}\right)$ satisfies

$$
\int_{\mathbb{H}^{n}} \frac{|f(z, t)| e^{\left(a^{p} / p\right)|t|^{p}}}{(1+|t|)^{d}} d(z, t)<\infty, \quad \int_{\mathbf{R}} \frac{\|\widehat{f}(\lambda)\|_{H S} e^{\left(b^{p} / q\right)|\lambda|^{q}}}{(1+|\lambda|)^{d}} d \mu(\lambda)<\infty,
$$

where $1<p, q<\infty,(1 / p)+(1 / q)=1$ and $a, b$ are positive numbers such that $a b \geqslant 1$. Then $f=0$ unless $p=q=2, a b=1$ and $d>(n / 2)+1$, in which case,

$$
f(z, t)=e^{-a t^{2}}\left(\sum_{j=0}^{m} \varphi_{j}(z) t^{j}\right),
$$

where $\varphi_{j}(z) \in L^{2}\left(\mathbb{C}^{n}\right)$ and $m<d-((n / 2)+1)$. In particular, $f(z, t)=e^{-a t^{2}} f(z, 0)$ when $d \leqslant(n / 2)+2$.

The lower bound 1 in Corollary 1 is not sharp for $p \neq 2$. The critical low bound should be $|\cos (p \pi / 2)|^{1 / p}$ as proved by Bonami, Demange and Jaming [4] on Euclidean spaces.

THEOREM 3. Let $d \geqslant 0$ and assume that $f \in L^{2}\left(\mathbb{H}^{n}\right)$ satisfies

$$
\begin{aligned}
& \int_{\mathbf{H}^{n}} \frac{|f(z, t)| e^{\left(a^{p} / p\right)|t|^{p}}}{(1+|t|)^{d}} d(z, t)<\infty, \\
& \int_{\mathbf{R}} \frac{\|\widehat{f}(\lambda)\|_{H S} e^{(6 / / q)|\lambda|^{q}}}{\left(1+\left.|\lambda|\right|^{d}\right.} d \mu(\lambda)<\infty,
\end{aligned}
$$

where $1<p<2,(1 / p)+(1 / q)=1$ and $a, b$ are positive numbers. Then $f=0$ if $a b>|\cos (p \pi / 2)|^{1 / p}$.

Proof: We can choose $\varepsilon>0$ such that $(a-\varepsilon)(b-\varepsilon)>|\cos (p \pi / 2)|^{1 / p}$. By same argument of Theorem 2 , for any $\alpha, \beta \in \mathbb{N}^{n}$, we get from (12) and (13) that

$$
\begin{aligned}
& \int_{\mathbf{R}}\left|g_{\alpha, \beta}(t)\right| e^{1 / p \alpha^{p}|t|^{p}} d t<\infty, \\
& \int_{\mathbf{R}}\left|\widehat{g_{\alpha, \beta}}(\lambda)\right| e^{1 / q b^{\alpha}|\lambda|^{q}} d \lambda<\infty,
\end{aligned}
$$

where $g_{\alpha, \beta}$ is defined by (6). It follows that

$$
\begin{aligned}
& \int_{\mathbf{R}}\left|g_{\alpha, \beta}(t)\right| e^{(1 / p)(a-\varepsilon)^{p}|t|^{p}} d t<\infty, \\
& \int_{\mathbf{R}}\left|\widehat{g_{\alpha, \beta}}(\lambda)\right| e^{1 / q(b-\varepsilon)^{q}|\lambda|^{q}} d \lambda<\infty .
\end{aligned}
$$

By [4, Theorem 1.4], $g_{\alpha, \beta}=0$. Theorem 3 is proved.

As a consequence of Theorem 3 , we have 
COROLlaRY 2. Let $d \geqslant 0$. Suppose $f \in L^{1}\left(\mathbb{H}^{n}\right) \cap L^{2}\left(\mathbb{H}^{n}\right)$ satisfies

$$
\int_{\mathbb{C}^{n}}|f(z, t)| d z \leqslant C(1+|t|)^{d} e^{-a|t| p}, \quad|\lambda|^{n / 2}\|\widehat{f}(\lambda)\|_{H S} \leqslant C(1+|\lambda|)^{d} e^{-b|\lambda| q},
$$

where $C>0,1<p<2,1 / p+1 / q=1$ and $a, b$ are positive numbers. Then $f=0$ if $a b>1 / 4|\cos (p \pi / 2)|^{2 / p}$.

Finally we list some known results which are easy to derive from above.

COROLlary 3. (Bagchi and Ray [3]) Suppose $f \in L^{1}\left(\mathbb{H}^{n}\right) \cap L^{2}\left(\mathbb{H}^{n}\right)$ satisfies

$$
\int_{\mathbb{H}^{n}}\left(|f(z, t)| e^{a\|(z, t)\|^{2}}\right)^{p} d(z, t)<\infty, \quad \int_{\mathbb{R}}\left(\|\widehat{f}(\lambda)\|_{H S} e^{b \lambda^{2}}\right)^{q} d \mu(\lambda)<\infty,
$$

where $\min (p, q)<\infty, q \geqslant 2, a, b$ are positive numbers and $\|(z, t)\|=\left(|z|^{2}+t^{2}\right)^{1 / 2}$ is the Euclidean norm of $(z, t) \in \mathbb{C}^{n} \times \mathbb{R}$. Then $f=0$ if $a b>1 / 4$.

COROLlary 4. (Bagchi and Ray [3]) Suppose $f$ is a measurable function on $\mathbb{H}^{n}$ which satisfies

$$
|f(z, t)| \leqslant C g(z) e^{-a|t| p}, \quad\|\widehat{f}(\lambda)\|_{H S} \leqslant C e^{-b|\lambda|^{q}},
$$

where $a, b, C>0, p \geqslant 2,1 / p+1 / q=1$ and $g \in L^{1}\left(\mathbb{C}^{n}\right) \cap L^{2}\left(\mathbb{C}^{n}\right)$. If $(a p)^{1 / p}(b q)^{1 / q}>1$, then $f=0$.

COROLlary 5. (Thangavelu [15]) Suppose $f$ is a measurable function on $\mathbb{H}^{n}$ which satisfies

$$
|f(z, t)| \leqslant C g(z) e^{-a t^{2}}, \quad|\lambda|^{n / 2}\|\widehat{f}(\lambda)\|_{H S} \leqslant C e^{-b \lambda^{2}},
$$

where $a, b, C>0$ and $g \in L^{1}\left(\mathbb{C}^{n}\right) \bigcap L^{2}\left(\mathbb{C}^{n}\right)$. If $a b>1 / 4$, then $f=0$. If $a b=1 / 4$, then $f(z, t)=e^{-a t^{2}} f(z, 0)$.

\section{REFERENCES}

[1] F. Astengo, M. Cowling, B. Di Blasio and M. Sundari, 'Hardy's uncertainty pronciple on some Lie groups', J. London Math. Soc. 62 (2000), 461-472.

[2] A. Baclouti and S. N. Ben, 'The $L^{p}-L^{q}$ version of Hardy's theorem on nilpotent Lie groups', Forum Math. 18 (2006), 245-262.

[3] S.C. Bagchi and S.K. Ray, 'Uncertainty principles like Hardy's theorem on some Lie groups', J. Aust. Math. Soc. Ser. A 65 (1999), 289-302.

[4] A. Bonami, B. Demange and P. Jaming, 'Hermite functions and uncertainty principles for the Fourier and the widowed Fourier transform', Rev. Mat. Iberoamericana 19 (2003), 23-55.

[5] G.B. Folland, Harmoic analysis in phase space, Ann. Math. Stud. 122 (Princeton University Press, Princeton, NJ, 1989).

[6] G.B. Folland and A. Sitaram, 'The uncertainty principle: a mathematical survey', $J$. Fourier Anol. Appl. 3 (1997), 207-238. 
[7] L. Hömander, 'A uniqueness theorem of Beurling for Fourier transform pairs', Ark. Mat. 29 (1991), 237-240.

[8] E. Kaniuth and A. Kumar, 'Hardy's theorem for simply connected nilpotent Lie groups', Math. Proc. Cambridge Philos. Soc. 131 (2001), 487-494..

[9] A. Kumar and C.R. Bhatta, 'An uncertainty principle like Hardy's theorem for nilpotent Lie groups', J. Aust. Math. Soc. 77 (2004), 47-53.

[10] S.K. Ray, Uncertainty principles on two step nilpotent Lie groups, Proc. Ind. Acad. Sci. Math. Soc. 111, pp. 293-318.

[11] R.P. Sarkar and J. Sengupta, 'Beurling's theorem and characterization of heat kernel for Riemannian symmtric spaces of noncompact type', arXiv: math. FA/0502514, V1, (2005).

[12] R.P. Sarkar and S. Thangavelu, 'On theorems of Beurling and Hardy for the Euclidean Motion group', Tohoku. Math. J. 57 (2005), 335-351.

[13] A. Sitaram, M. Sundari and S. Thangavelu, 'Uncertainty principles on certain Lie groups', Proc. Indian Acad. Sci. Math. Sci. 105 (1995), 135-151.

[14] S. Thangavelu, Lectures on Hermite and Laguerre expansions, Math. Notes 42 (Princeton University Press, Princeton, NJ, 1993).

[15] S. Tangavelu, Harmonic analysis on the Heisenberg group, Progr. Math. 159 (Birkhäuser, Boston, 1998).

[16] S. Thangavelu, 'An analogue of Hardy's theorem for the Heisenberg group', Colloq. Math. 87 (2001), 137-145.

[17] S. Thangavelu, 'Revisiting Hardy's theorem for the Heisenberg group', Math. Z. 242 (2002), 761-779.

[18] S. Thangavelu, An introduction to the uncertainty principle, Progr. Math. 217 (Birkhäuser, Boston, 2003).

[19] S. Thangavelu, 'On theorems of Hardy, Gelfand-Shilov and Beurling for semisimple Lie groups', Publ. Res. Inst. Math. Sci. 40 (2004), 311-344..

LMAM, School of Mathematical Sciences

Peking University

Beijing 100871

People's Republic of China

e-mail: hjzheng@pku.edu.cn; hpliu@pku.edu.cn 